Supporting information for

\title{
Atomistic modeling of PEDOT:PSS complexes I: DFT benchmarking
}

\author{
Wesley Michaels, ${ }^{*} \dagger$ Yan Zhao, ${ }^{\ddagger}$ and and Jian Qin, ${ }^{*, \dagger}$ \\ $\dagger$ Department of Chemical Engineering, Stanford University, Stanford, CA 94305 \\ $\ddagger$ State Key Laboratory of Silicate Materials for Architectures, Wuhan University of \\ Technology, Wuhan 430070, China
}

E-mail: wpm216@stanford.edu; jianq@stanford.edu

\section{Summary of Tables and Figures}

Table S1: Spin contamination of ab initio and two DFT methods for open-shell $\mathrm{PEDOT}_{3}{ }^{+}$, before and after contaminant annihilation.

Table S2: Mean absolute error of geometric benchmark measures.

Table [S3: Best approximations to ground-state geometries of selected complexes at DSDPBEP86 level.

Figure [S1: BLA in $\mathrm{PEDOT}_{3}$ and $\mathrm{PEDOT}_{3}{ }^{+}$, complementary to Figure 2.

Figure S2: Full fractional electron $\Delta E(\Delta N)$ curves.

Table S4: Energetic and geometric measures of EDOT ‥EDOT complexation.

Table S5: Polarizability tensor eigenvalues for molecules studied.

Table S6: Performance of B3LYP and DSD-PBEP86 in benchmark by Winter and Falvey. 108

Table S7: SP energies of PEDOT 6 at DF-DSD-PBEP86/jun-cc-pV $(\mathrm{T}+\mathrm{d}) \mathrm{Z} / /\{\mathrm{X}\}$ level.

Figure [33: Full torsional profiles for bipolaronic and pair-polaronic $\mathrm{PEDOT}_{6}{ }^{2+}$.

Table [S8: $\phi_{c}$ values for DFs studied.

Table [S9; Full benchmarking rankings for all DFs studied. 


\section{Supporting Information}

Table S1: Table of $\mathrm{PEDOT}_{3}{ }^{+}$spin contamination for HF, MP2, PBE0, and DSD-PBEP86. $S^{2}$ and $S_{\mathrm{A}}^{2}$ represent total spin squared and annihilated spin squared, respectively.

\begin{tabular}{|c|c|c|c|}
\hline $\mathrm{LC}$ & Method & $\mathrm{PEDOT}_{3}+S^{2}$ & $\mathrm{PEDOT}_{3}+S_{\mathrm{A}}^{2}$ \\
\hline \multirow{2}{*}{ (ab initio) } & $\mathrm{HF}$ & 1.42 & 1.24 \\
\hline & MP2 & 1.42 & 1.24 \\
\hline \multirow{12}{*}{ No LC } & DSD-PBEP86 & 0.981 & 0.794 \\
\hline & APFD & 0.778 & 0.751 \\
\hline & B3LYP & 0.772 & 0.750 \\
\hline & B3LYP-D3 & 0.772 & 0.750 \\
\hline & B97-D & 0.757 & 0.750 \\
\hline & B97-D3 & 0.757 & 0.750 \\
\hline & HSE06 & 0.777 & 0.751 \\
\hline & M062-X & 0.786 & 0.751 \\
\hline & M06-HF & 0.822 & 0.753 \\
\hline & M06-L & 0.761 & 0.750 \\
\hline & MN15 & 0.781 & 0.751 \\
\hline & PBE0 & 0.781 & 0.751 \\
\hline \multirow{6}{*}{$\mathrm{LC}$} & CAM-B3LYP & 0.816 & 0.753 \\
\hline & CAM-B3LYP-D3 & 0.816 & 0.753 \\
\hline & LC-BLYP & 0.887 & 0.763 \\
\hline & LC- $\omega \mathrm{HPBE}$ & 0.882 & 0.762 \\
\hline & LC- $\omega$ PBE-D3 & 0.883 & 0.762 \\
\hline & $\omega \mathrm{B} 97 \mathrm{x}-\mathrm{D}$ & 0.813 & 0.752 \\
\hline
\end{tabular}


Table S2: $\operatorname{MAE}\left(\Delta\left|Z_{i}\right|\right)$ of geometric benchmark measures.

\begin{tabular}{cllll} 
LC & Functional & Intramolecular & Intermolecular & Aggregate \\
\hline \hline \multirow{4}{*}{ No LC } & APFD & 0.50 & 2.60 & 0.97 \\
& B3LYP & 0.32 & 1.59 & 0.60 \\
& B3LYP-D3 & 0.33 & 0.85 & 0.44 \\
& B97-D & 0.94 & 2.68 & 1.33 \\
& HSE06 & 0.73 & 0.47 & 0.67 \\
& M062-X & 0.57 & 1.19 & 0.71 \\
& M06-HF & 0.87 & 0.21 & 0.53 \\
& M06-L & 0.51 & 10.30 & 2.97 \\
& MN15 & 0.66 & 4.63 & 1.42 \\
& PBE0 & 0.61 & 0.31 & 0.58 \\
\hline \multirow{4}{*}{ LC } & CAM-B3LYP & 0.56 & 0.82 & 0.72 \\
& CAM-B3LYP-D3 & 0.56 & 1.20 & 0.62 \\
& LC-BLYP & 1.29 & 3.92 & 1.88 \\
& LC- $\omega$ HPBE & 0.93 & 0.76 & 0.89 \\
& LC- $\omega$ PBE-D3 & 0.91 & 1.24 & 0.99 \\
& $\omega$ B97x-D & 0.59 & 1.16 & 0.72 \\
\hline
\end{tabular}

Table S3: Best approximations to select DSD-PBEP86 ground-state geometries.

\begin{tabular}{ccc} 
Complex & Best geometry & Energy (Hartrees) \\
\hline \hline PEDOT $_{3}{ }^{+} \ldots$ TFSI $^{-}$ & LC-BLYP/jun-cc-pVDZ & -4159.355988 \\
PEDOT $_{3}{ }^{+} \ldots$ TOS $^{-}$ & LC-BLYP/jun-cc-pVDZ & -3267.355905 \\
\hline
\end{tabular}


(a)

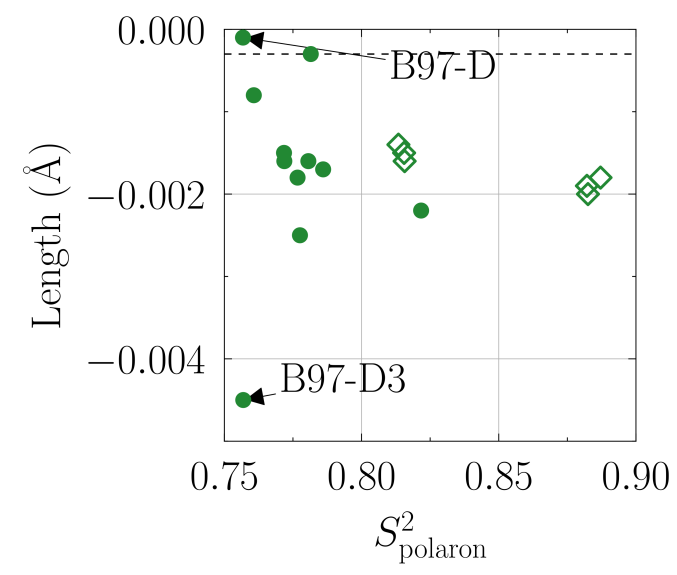

(b)

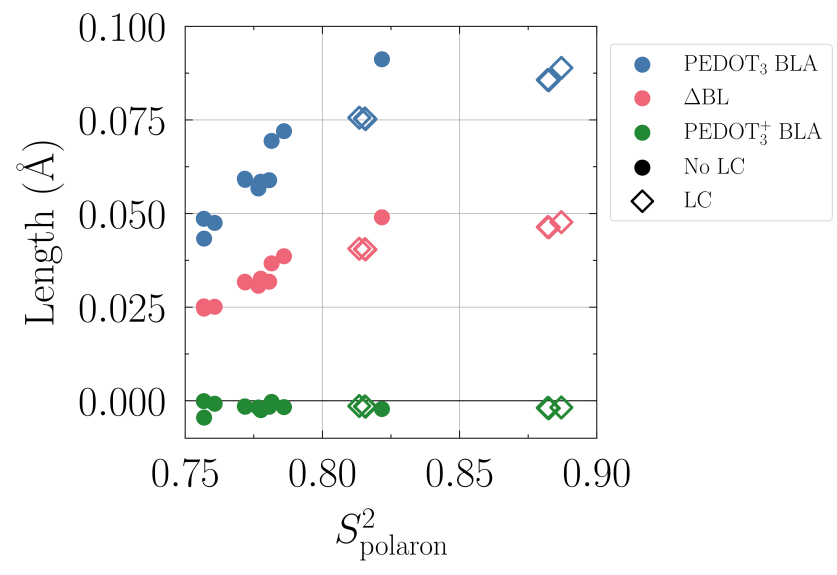

Figure S1: Full detail of BLA in $\mathrm{PEDOT}_{3}$ and $\mathrm{PEDOT}_{3}{ }^{+}$(complementary to Figure 2) . (a) BLA in $\mathrm{PEDOT}_{3}{ }^{+}$. B97-D and B97-D3 results annotated for clarity. (b) Data in (a) shown along with BLA in $\mathrm{PEDOT}_{3}$ and $\triangle \mathrm{BL}$.

(a)

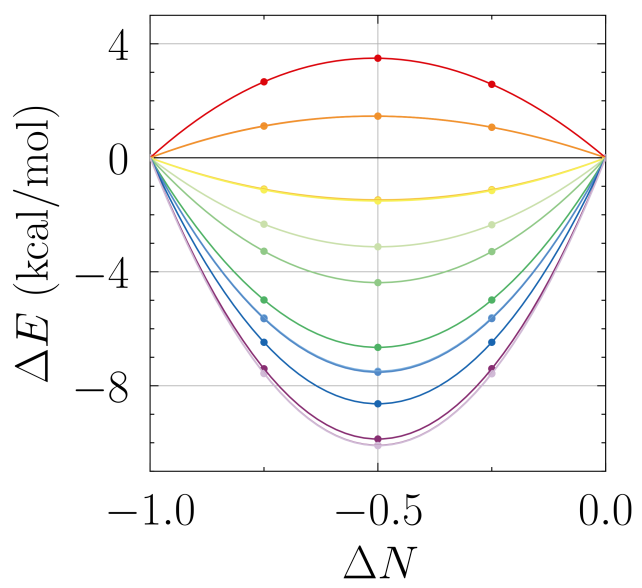

(b)

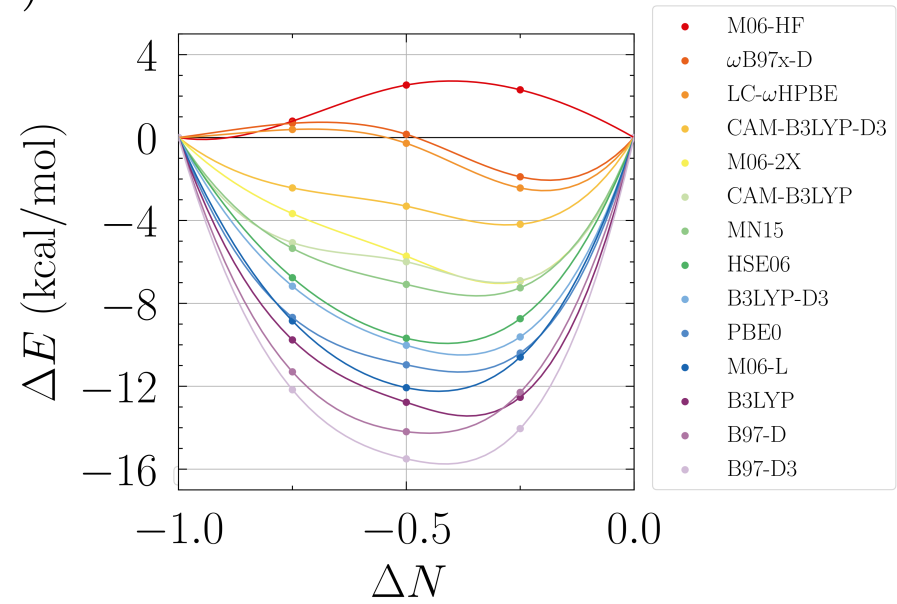

Figure S2: Full fractional electron curves for DFs available in Psi4. (a) $\Delta E$ vs $\Delta N$ with all points evaluated at ground-state $\mathrm{PEDOT}_{3}$ geometries. Some functionals overlap quite closely: $\omega$ B97x-D and LC $\omega$ HPBE; CAM-B3LYP-D3 and M06-2X; and B97-D and B97-D3. (a) $\Delta E$ vs $\Delta N$ with geometry optimized for each point. Data are points; splines are added to guide the eye. APFD, LC $\omega$ PBE-D3, and LC-BLYP were not available in Psi4 and their results are therefore not shown. 
Table S4: Energetic and geometric measures of EDOT $\cdots$ EDOT interaction. $\Delta E=E_{\text {skewed }}-$ $E_{\text {antiparallel }}$. Other measures are defined in Figure 3 .

\begin{tabular}{|c|c|c|c|c|c|c|c|c|}
\hline Geometry & $\mathrm{LC}$ & Functional & $\Delta E(\mathrm{kcal} / \mathrm{mol})$ & $d_{\mathrm{t}}(\AA)$ & $d_{\mathrm{v}}(\AA)$ & $d_{\mathrm{h}}(\AA)$ & $\theta_{\text {ip }}$ (deg.) & $\theta_{\mathrm{r}}$ (deg.) \\
\hline \multirow{18}{*}{ Skewed } & \multirow{12}{*}{ No LC } & DSD-PBEP86 & -2.58 & 3.48 & 3.38 & 0.85 & 178.7 & 68.6 \\
\hline & & APFD & -3.07 & 3.46 & 3.37 & 0.81 & 179.7 & 68.6 \\
\hline & & B3LYP & -0.61 & 8.23 & 2.73 & 7.77 & 57.8 & 170.7 \\
\hline & & B3LYP-D3 & 0.32 & 3.64 & 3.53 & 0.86 & 177.9 & 66.0 \\
\hline & & B97-D & 1.06 & 3.66 & 3.54 & 0.93 & 178.2 & 70.4 \\
\hline & & B97-D3 & 0.55 & 3.62 & 3.52 & 0.86 & 176.9 & 67.7 \\
\hline & & HSE06 & 0.98 & 4.02 & 3.90 & 0.94 & 179.7 & 64.5 \\
\hline & & M06-2X & -2.62 & 3.50 & 3.40 & 0.82 & 179.7 & 69.5 \\
\hline & & M06-HF & -2.93 & 3.48 & 3.37 & 0.87 & 178.1 & 66.4 \\
\hline & & M06-L & -2.04 & 3.53 & 3.44 & 0.77 & 178.9 & 72.1 \\
\hline & & MN15 & -2.19 & 3.56 & 3.47 & 0.79 & 179.7 & 70.7 \\
\hline & & PBE0 & 0.95 & 4.03 & 3.92 & 0.94 & 179.4 & 64.7 \\
\hline & \multirow{6}{*}{$\mathrm{LC}$} & CAM-B3LYP & -0.57 & 6.43 & 4.62 & 4.47 & 112.0 & 125.4 \\
\hline & & CAM-B3LYP-D3 & 0.23 & 3.63 & 3.53 & 0.86 & 178.4 & 65.4 \\
\hline & & LC-BLYP & 0.10 & 3.75 & 3.65 & 0.86 & 180.0 & 63.9 \\
\hline & & LC- $\omega \mathrm{HPBE}$ & 0.56 & 3.89 & 3.79 & 0.86 & 178.6 & 64.2 \\
\hline & & LC- $\omega$ PBE-D3 & -2.56 & 3.53 & 3.43 & 0.81 & 177.7 & 67.6 \\
\hline & & $\omega \mathrm{B} 97 \mathrm{x}-\mathrm{D}$ & -2.80 & 3.63 & 3.53 & 0.83 & 179.5 & 65.8 \\
\hline \multirow{18}{*}{ Antiparallel } & \multirow{12}{*}{ No LC } & DSD-PBEP86 & - & 3.53 & 3.48 & 0.60 & 168.8 & 152.4 \\
\hline & & APFD & - & 3.53 & 3.46 & 0.67 & 170.7 & 152.5 \\
\hline & & B3LYP & - & 5.42 & 4.44 & 3.11 & 177.7 & 164.5 \\
\hline & & B3LYP-D3 & - & 4.76 & 3.66 & 3.04 & 152.5 & 178.7 \\
\hline & & B97-D & - & 4.80 & 3.68 & 3.08 & 156.5 & 179.6 \\
\hline & & B97-D3 & - & 4.76 & 3.71 & 2.98 & 156.0 & 178.3 \\
\hline & & HSE06 & - & 5.02 & 4.08 & 2.92 & 168.4 & 173.8 \\
\hline & & M06-2X & - & 3.60 & 3.49 & 0.87 & 171.4 & 156.8 \\
\hline & & M06-HF & & 3.56 & 3.44 & 0.93 & 170.8 & 157.5 \\
\hline & & M06-L & - & 3.66 & 3.52 & 0.98 & 174.7 & 168.0 \\
\hline & & MN15 & - & 3.67 & 3.58 & 0.83 & 169.6 & 153.1 \\
\hline & & PBE0 & - & 5.03 & 4.09 & 2.93 & 167.3 & 174.7 \\
\hline & \multirow{6}{*}{$\mathrm{LC}$} & CAM-B3LYP & - & 5.18 & 4.24 & 2.99 & 179.1 & 164.0 \\
\hline & & CAM-B3LYP-D3 & - & 4.74 & 3.65 & 3.02 & 151.6 & 179.7 \\
\hline & & LC-BLYP & - & 4.78 & 3.74 & 2.97 & 152.9 & 180.0 \\
\hline & & LC- $\omega \mathrm{HPBE}$ & - & 4.93 & 3.97 & 2.92 & 159.8 & 179.0 \\
\hline & & LC- $\omega$ PBE-D3 & - & 3.60 & 3.51 & 0.80 & 171.2 & 156.8 \\
\hline & & $\omega \mathrm{B} 97 \mathrm{x}-\mathrm{D}$ & - & 3.71 & 3.67 & 0.53 & 168.8 & 155.6 \\
\hline
\end{tabular}


Table S5: Polarizability tensor eigenvalues (Bohr) for all molecules studied with the exception of the open-shell $\mathrm{PEDOT}_{3}{ }^{+}$, calculations of which at the DSD-PBEP86/aug-cc-pVDZ level of theory exceeded computational capabilities.

\begin{tabular}{|c|c|c|c|c|c|c|c|c|c|c|}
\hline \multirow[b]{2}{*}{$\mathrm{LC}$} & \multirow[b]{2}{*}{ Functional } & \multicolumn{3}{|c|}{ EDOT } & \multicolumn{3}{|c|}{$\underline{\mathrm{PEDOT}_{3}}$} & \multicolumn{3}{|c|}{$\underline{\text { TOS }}$} \\
\hline & & $\alpha_{1}$ & $\alpha_{2}$ & $\alpha_{3}$ & $\alpha_{1}$ & $\alpha_{2}$ & $\alpha_{3}$ & $\alpha_{1}$ & $\alpha_{2}$ & $\alpha_{3}$ \\
\hline \multirow{12}{*}{ No LC } & DSD-PBEP86 & 93.0 & 66.2 & 129.3 & 467.7 & 171.1 & 358.5 & 186.8 & 101.8 & 131.2 \\
\hline & APFD & 87.3 & 60.9 & 122.7 & 476.0 & 161.5 & 347.8 & 177.0 & 93.7 & 124.4 \\
\hline & B3LYP & 89.0 & 62.2 & 125.4 & 491.0 & 164.8 & 356.4 & 182.2 & 96.1 & 127.2 \\
\hline & B3LYP-D3 & 89.0 & 62.3 & 125.4 & 491.4 & 164.8 & 356.2 & 181.8 & 96.2 & 127.2 \\
\hline & B97-D & 91.9 & 63.7 & 129.9 & 540.2 & 168.8 & 371.7 & 196.2 & 100.5 & 132.3 \\
\hline & B97-D3 & 91.6 & 63.6 & 129.8 & 540.3 & 168.5 & 371.2 & 195.1 & 100.1 & 131.8 \\
\hline & HSE06 & 87.3 & 61.2 & 122.7 & 480.1 & 162.2 & 348.5 & 177.4 & 94.0 & 124.6 \\
\hline & M06-2X & 86.0 & 60.4 & 120.3 & 433.4 & 160.8 & 339.8 & 169.0 & 91.2 & 122.0 \\
\hline & M06-HF & 85.2 & 61.0 & 118.0 & 391.6 & 161.5 & 331.2 & 166.5 & 91.4 & 120.5 \\
\hline & M06-L & 85.8 & 58.8 & 122.7 & 504.3 & 157.9 & 349.9 & 175.8 & 90.6 & 121.4 \\
\hline & MN15 & 86.8 & 61.1 & 121.0 & 447.6 & 162.5 & 343.2 & 173.4 & 93.9 & 124.7 \\
\hline & PBE0 & 87.1 & 61.0 & 122.3 & 472.3 & 161.6 & 347.3 & 176.3 & 93.5 & 124.2 \\
\hline \multirow{6}{*}{$\mathrm{LC}$} & CAM-B3LYP & 86.6 & 61.0 & 121.6 & 433.7 & 161.6 & 343.7 & 171.2 & 93.2 & 123.6 \\
\hline & CAM-B3LYP-D3 & 86.6 & 61.0 & 121.6 & 433.8 & 161.7 & 343.6 & 171.0 & 93.2 & 123.6 \\
\hline & LC-BLYP & 83.3 & 59.1 & 117.1 & 385.6 & 157.1 & 329.6 & 160.0 & 89.3 & 119.2 \\
\hline & LC-wHPBE & 83.3 & 117.7 & 58.5 & 391.7 & 156.0 & 332.2 & 162.1 & 89.5 & 119.5 \\
\hline & LC-wPBE-D3 & 83.4 & 58.5 & 117.7 & 391.9 & 156.0 & 332.1 & 161.9 & 89.5 & 119.5 \\
\hline & wB97x-D & 86.1 & 60.7 & 120.8 & 429.0 & 161.1 & 341.5 & 170.0 & 92.8 & 123.0 \\
\hline \multirow[b]{2}{*}{$\mathrm{LC}$} & & \multicolumn{3}{|c|}{$\underline{\text { TOS-H }}$} & \multicolumn{3}{|c|}{$\underline{\text { EMIM }^{+}}$} & \multicolumn{3}{|c|}{$\underline{\text { TFSI }^{-}}$} \\
\hline & Functional & $\alpha_{1}$ & $\alpha_{2}$ & $\alpha_{3}$ & $\alpha_{1}$ & $\alpha_{2}$ & $\alpha_{3}$ & $\alpha_{1}$ & $\alpha_{2}$ & $\alpha_{3}$ \\
\hline \multirow{12}{*}{ No LC } & DSD-PBEP86 & 174.7 & 93.4 & 123.5 & 102.9 & 79.5 & 59.1 & 122.8 & 92.8 & 96.1 \\
\hline & APFD & 165.1 & 85.8 & 117.7 & 98.2 & 75.1 & 55.0 & 111.8 & 85.0 & 86.8 \\
\hline & B3LYP & 170.1 & 87.5 & 119.6 & 100.0 & 76.2 & 55.7 & 117.7 & 87.9 & 90.7 \\
\hline & B3LYP-D3 & 169.7 & 87.6 & 119.6 & 99.8 & 76.2 & 55.7 & 117.2 & 87.9 & 90.6 \\
\hline & B97-D & 179.1 & 90.9 & 123.2 & 103.1 & 77.9 & 56.8 & 129.0 & 97.9 & 93.2 \\
\hline & B97-D3 & 178.0 & 90.4 & 122.9 & 102.9 & 77.8 & 56.7 & 127.3 & 92.7 & 96.6 \\
\hline & HSE06 & 165.6 & 85.8 & 117.8 & 98.5 & 75.2 & 55.0 & 112.3 & 85.2 & 86.8 \\
\hline & M06-2X & 160.2 & 84.2 & 116.3 & 97.4 & 74.9 & 54.9 & 106.5 & 81.7 & 83.0 \\
\hline & M06-HF & 159.0 & 84.2 & 114.9 & 95.0 & 73.1 & 55.4 & 104.7 & 83.6 & 80.8 \\
\hline & M06-L & 164.5 & 84.2 & 115.8 & 99.0 & 74.7 & 53.7 & 112.1 & 83.5 & 84.6 \\
\hline & MN15 & 163.3 & 85.5 & 118.3 & 98.9 & 75.7 & 55.4 & 107.6 & 83.2 & 84.0 \\
\hline & PBE0 & 164.9 & 85.5 & 117.5 & 98.3 & 75.1 & 55.0 & 111.6 & 84.8 & 86.4 \\
\hline \multirow{6}{*}{$\mathrm{LC}$} & CAM-B3LYP & 162.4 & 85.1 & 117.1 & 97.2 & 74.9 & 54.9 & 109.8 & 84.8 & 86.2 \\
\hline & CAM-B3LYP-D3 & 162.1 & 85.1 & 117.1 & 97.2 & 74.8 & 54.9 & 109.8 & 84.8 & 86.2 \\
\hline & LC-BLYP & 153.4 & 81.8 & 113.8 & 93.9 & 72.9 & 53.7 & 102.7 & 81.3 & 81.4 \\
\hline & LC-wHPBE & 154.7 & 82.1 & 114.2 & 94.0 & 72.8 & 53.4 & 105.3 & 82.7 & 82.8 \\
\hline & LC-wPBE-D3 & 154.4 & 82.2 & 114.2 & 93.9 & 72.7 & 53.4 & 105.1 & 82.7 & 82.8 \\
\hline & wB97x-D & 161.5 & 84.8 & 116.6 & 96.5 & 74.6 & 54.9 & 109.0 & 83.9 & 85.2 \\
\hline
\end{tabular}


Table S6: Performance of various DFs in the $\Delta E_{\mathrm{ST}}$ benchmark by Winter and Falvey. 108 Results shown are SP energies at CASSCF/cc-pVTZ geometries unless otherwise noted. ${ }^{\dagger}$ Geometries optimized at B3LYP/6-31G(d,p) level of theory. ${ }^{a}$ By Winter and Falvey. 108 ${ }^{b}$ Calculated in this work.

Functional

\begin{tabular}{ccccc}
\cline { 2 - 5 } Structure $^{a}$ & CASPT2 $^{a}$ & ref $(\mathrm{B} 3 \mathrm{LYP})^{a \dagger}$ & B3LYP $^{b}$ & DSD-PBEP86 $^{b}$ \\
\hline \hline 2 & -53.2 & -46.2 & -51.7 & -50.2 \\
3 & -51.6 & -46.4 & -47.8 & -48.2 \\
4 & -33 & -31.6 & -31.2 & -30.8 \\
11 & -36.8 & -31.4 & -32.5 & -33.5 \\
13 & -26.5 & -20.2 & -20.6 & -22.3 \\
16 & -3.3 & -2.4 & -3.8 & -0.9 \\
17 & -5.6 & -3.1 & -3.7 & -3.8 \\
19 & 0.3 & 3.8 & 2.4 & 5.2 \\
21 & 5.8 & 4.6 & 2.7 & 5.8 \\
22 & 9.9 & 5.5 & 11.4 & 8.8 \\
23 & 11.9 & 11.1 & 11.7 & 14.1 \\
24 & 14.1 & 17.1 & 15.1 & 17.8 \\
\hline MSE & - & 2.4 & 1.7 & 2.5 \\
MAE & - & 2.7 & 1.6 & 1.3 \\
\hline \hline
\end{tabular}


Table S7: PEDOT $_{6}$ DF-DSD-PBEP86/jun-cc-pV(T+d)Z SP energies at ground state geometries of DFs studied. Ground state, bipolaron, and polaron pair energy values are referenced to the minimum absolute energy in each column.

\begin{tabular}{|c|c|c|c|c|c|}
\hline \multirow[b]{2}{*}{$\mathrm{LC}$} & \multirow[b]{2}{*}{ Functional } & \multicolumn{4}{|c|}{$\mathrm{PEDOT}_{6}$ Energy $(\mathrm{kcal} / \mathrm{mol})$} \\
\hline & & Ground State & Bipolaron & Polaron pair & Average \\
\hline \multirow{11}{*}{ No LC } & APFD & 0.48 & 0.20 & 0.85 & 0.51 \\
\hline & B3LYP & 5.21 & 5.23 & 6.39 & 5.61 \\
\hline & B3LYP-D3 & 5.16 & 5.14 & 6.37 & 5.56 \\
\hline & B97-D & 13.09 & 13.02 & 14.95 & 13.69 \\
\hline & B97-D3 & 11.01 & 10.32 & 12.20 & 11.18 \\
\hline & HSE06 & 0.14 & 0.00 & 0.65 & 0.26 \\
\hline & M062-X & 0.00 & 0.41 & 0.40 & 0.27 \\
\hline & M06-HF & 2.19 & 3.47 & 1.70 & 2.45 \\
\hline & M06-L & 0.62 & 0.27 & 1.07 & 0.65 \\
\hline & MN15 & 0.13 & 0.34 & 0.47 & 0.31 \\
\hline & PBE0 & 0.03 & 0.03 & 0.60 & 0.22 \\
\hline \multirow{6}{*}{$\mathrm{LC}$} & CAM-B3LYP & 0.85 & 1.44 & 1.22 & 1.17 \\
\hline & CAM-B3LYP-D3 & 0.82 & 1.42 & 1.28 & 1.17 \\
\hline & LC-BLYP & 1.88 & 3.07 & 1.05 & 2.00 \\
\hline & LC- $\omega \mathrm{HPBE}$ & 0.36 & 1.37 & 0.01 & 0.58 \\
\hline & LC- $\omega$ PBE-D3 & 0.35 & 1.30 & 0.00 & 0.55 \\
\hline & $\omega \mathrm{B} 97 \mathrm{x}-\mathrm{D}$ & 0.75 & 1.48 & 1.14 & 1.12 \\
\hline
\end{tabular}


(a)

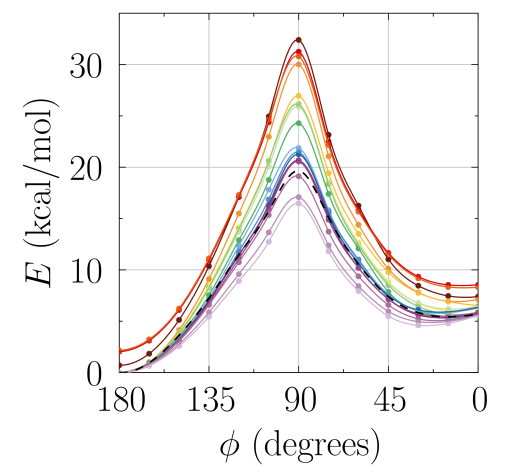

(b)

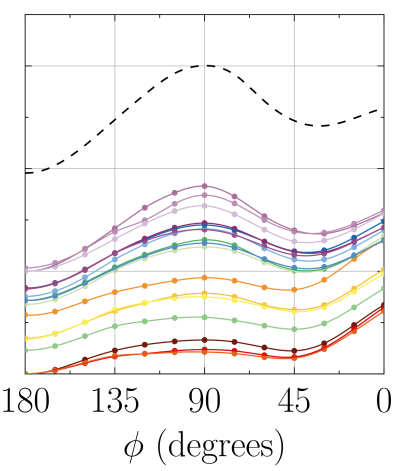

(c)

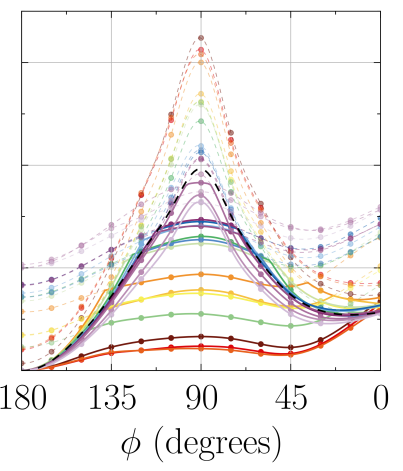

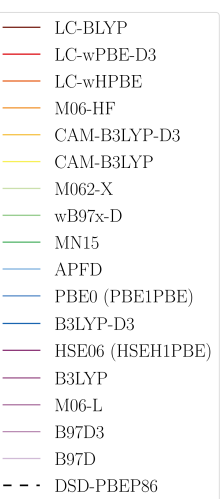

Figure S3: Full torsion profiles for $\mathrm{PEDOT}_{6}{ }^{2+}$ about middle inter-mononmer bond. (a) Bipolaronic and (b) pair-polaronic spin states. (c) Aggregation of (a) and (b): (solid lines) minimum-energy profiles incorporating both spin states and (dashed line) maximum-energy profiles. 
Table S8: $\phi_{c}$ values for DFs studied, defined as the $\mathrm{PEDOT}_{6}{ }^{2+}$ torsional angle at which the pair-polaronic state becomes more energetically favorable than the bipolaronic state.

\begin{tabular}{|c|c|c|}
\hline $\mathrm{LC}$ & Functional & $\phi_{\mathrm{c}}$ \\
\hline \multirow{11}{*}{ No LC } & APFD & $61^{\circ}$ \\
\hline & B3LYP & $68^{\circ}$ \\
\hline & B3LYP-D3 & $68^{\circ}$ \\
\hline & B97-D & $88^{\circ}$ \\
\hline & B97-D3 & - \\
\hline & HSE06 & $68^{\circ}$ \\
\hline & M062-X & $53^{\circ}$ \\
\hline & M06-HF & $42^{\circ}$ \\
\hline & M06-L & $84^{\circ}$ \\
\hline & MN15 & $56^{\circ}$ \\
\hline & PBE0 & $60^{\circ}$ \\
\hline \multirow{6}{*}{$\mathrm{LC}$} & CAM-B3LYP & $36^{\circ}$ \\
\hline & CAM-B3LYP-D3 & $36^{\circ}$ \\
\hline & LC-BLYP & $0^{\circ}$ \\
\hline & LC- $\omega \mathrm{HPBE}$ & $0^{\circ}$ \\
\hline & LC- $\omega$ PBE-D3 & $0^{\circ}$ \\
\hline & $\omega B 97 x-D$ & $29^{\circ}$ \\
\hline
\end{tabular}




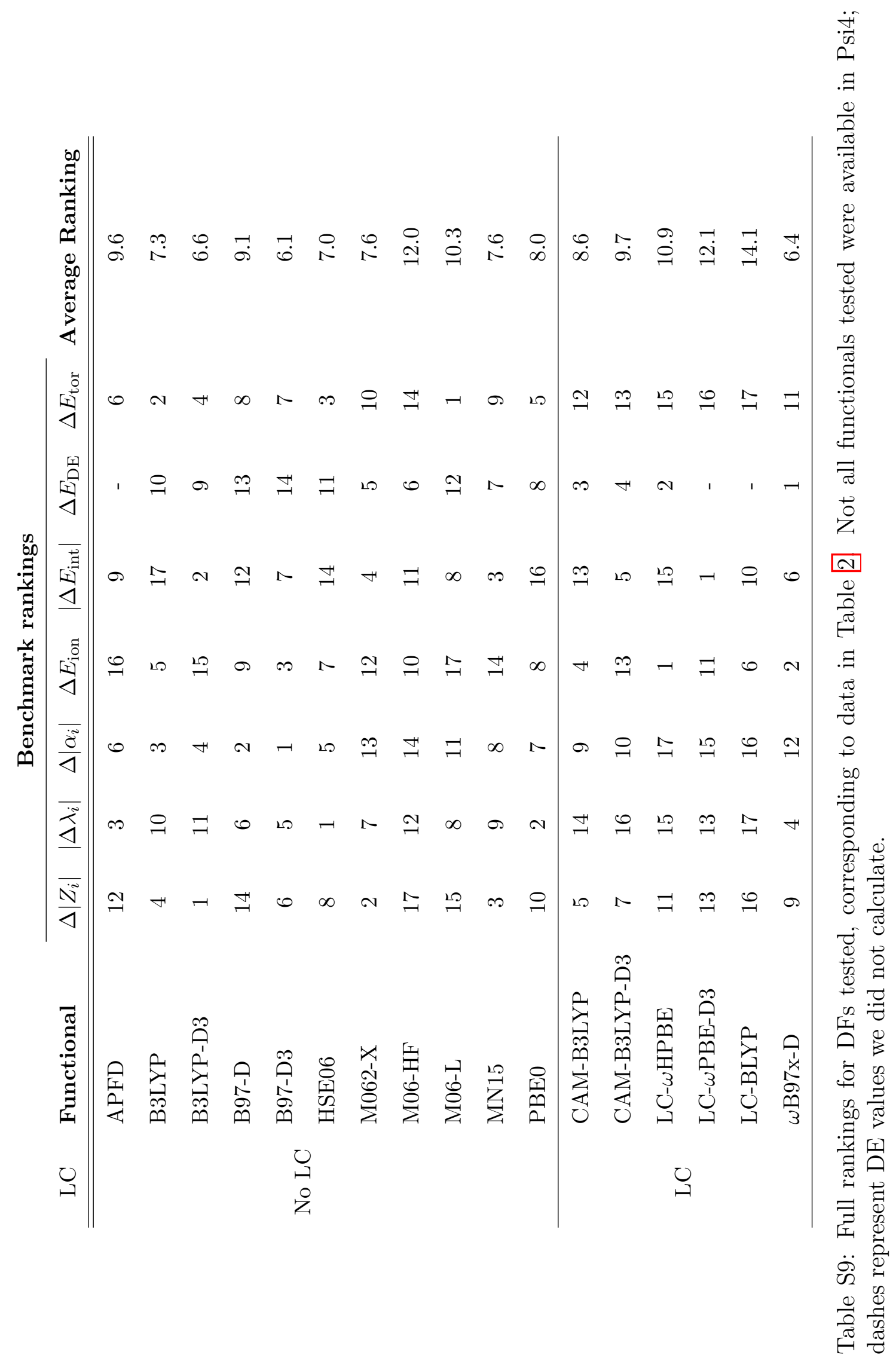

